\title{
La privatisation de l'éducation dans le système scolaire anglais
}

Privatization of education in the English school system

La privatización de la educación en el sistema escolar inglés

\section{Anne West}

Traducteur : Thierry Chevaillier

\section{(2) OpenEdition}

Journals

Édition électronique

URL : https://journals.openedition.org/ries/9145

DOI : 10.4000/ries. 9145

ISSN : 2261-4265

Éditeur

France Education international

Édition imprimée

Date de publication : 1 décembre 2019

Pagination : 76-84

ISBN : 9782854206258

ISSN : $1254-4590$

Référence électronique

Anne West, "La privatisation de l'éducation dans le système scolaire anglais », Revue internationale d'éducation de Sèvres [En ligne], 82 | décembre 2019, mis en ligne le 01 décembre 2021, consulté le 14 janvier 2022. URL : http://journals.openedition.org/ries/9145; DOI : https://doi.org/10.4000/ries.9145 


\title{
La privatisation de l'éducation dans le système scolaire anglais*
}

\author{
Anne West \\ London School of Economics
}

\section{LES MULTIPLES DIMENSIONS DE LA PRIVATISATION}

En Angleterre, le secteur privé intervient de manière significative dans l'enseignement scolaire financé par l'État. Depuis 1997, sous des majorités aussi bien travaillistes que conservatrices, le gouvernement a cherché à faire participer à la fourniture de services publics des acteurs privés, qu'ils soient à but lucratif ou non lucratif. L'un des principaux services publics visés a été l'éducation et les organismes privés sont intervenus de manière croissante dans l'enseignement scolaire, dans ce qui apparaît clairement comme une " privatisation ».

Cet article examine toute une gamme de mesures de politique qui ont concerné l'enseignement scolaire en Angleterre. On peut considérer qu'elles relèvent des profonds changements qui ont affecté, dans les années récentes, la gestion des services publics au Royaume-Uni et dans d'autres pays industrialisés, changements qui consistaient à utiliser dans le secteur public des concepts et des pratiques relevant du secteur privé.

La notion de privatisation fait l'objet d'un débat et ses définitions sont variées. Selon Whitfield (2006), il y a privatisation lorsque l'on modifie l'un ou plusieurs des cinq éléments constitutifs des services publics :

- la propriété des actifs publics (services, organisations, terrains, bâtiments, équipement, données et droits intellectuels) est vendue ou transférée au secteur privé ou au secteur associatif (privé sans but lucratif);

- le contrôle exercé sur les organismes publics est atténué ou dilué par le recours à de nouvelles structures d'organisation de type entreprise ou fondation ;

- les modes de financement sont modifiés et il est, notamment, fait appel aux capitaux privés ;

- les principes de fonctionnement et le système de valeurs se transforment pour aligner la fourniture de services publics sur le modèle de l'entreprise privée ;

- les organes de direction des organismes publics sont restructurés pour permettre la mise en œuvre de ces changements.

On trouve dans le système scolaire anglais des exemples de chacun de ces différents modes de privatisation. En premier lieu, des écoles relevant des autorités locales ont été « converties » en « academies » contrôlées et gérées par des «trusts »,

\footnotetext{
* Article traduit par Thierry Chevaillier.
} 
fondations privées sans but lucratif qui relèvent du droit des entreprises et opèrent selon les règles du secteur privé. En second lieu, on a recouru à des financements privés pour construire des écoles et les faire fonctionner sur de longues périodes dans le cadre de l'Initiative de financement privé, qui promeut les partenariats publicprivé. Troisièmement, les écoles, et pas seulement les academies mais aussi celles qui sont restées sous le contrôle des autorités locales, externalisent certaines de leurs activités, en les sous-traitant à l'extérieur. Ces réformes ont abouti à une réduction considérable du rôle des autorités locales dans l'enseignement scolaire.

Cet article montre que, dans le contexte anglais, la privatisation de l'enseignement soulève des problèmes en ce qui concerne le devoir de rendre des comptes au public, les coûts pour la collectivité et la transparence de l'information due au public. Après une brève présentation de l'évolution du système éducatif, les sections suivantes analysent, en s'appuyant sur la littérature existante et sur l'étude des documents publiés, les trois types de privatisation : les academies, l'externalisation et les partenariats public-privé. Une section finale discute les principaux sujets d'inquiétude que soulève la privatisation de l'enseignement : le contrôle démocratique, le coût pour les deniers publics et la transparence et l'ouverture au public des processus impliqués.

\section{LE SYSTÈME SCOLAIRE ANGLAIS}

La loi sur l'éducation de 1944 établissait un système national d'enseignement primaire et secondaire financé par l'État, constitué d'écoles fonctionnant sous la tutelle des autorités locales. L'autorité locale était responsable du financement et de la gestion des écoles (maintained schools). Il existait parallèlement un enseignement privé dans des écoles financées par les droits d'inscription versés par les parents. Les deux secteurs coexistent toujours à l'heure actuelle : $93 \%$ des élèves soumis à la scolarité obligatoire se trouvent aujourd'hui dans les écoles financées par l'État, le reste étant inscrit dans les écoles payantes, dont font partie les établissements d'élite, improprement appelés "public schools ", tels que Eton et Harrow.

Les coûts des établissements d'enseignement financés par l'État sont couverts par une dotation du gouvernement central. Jusqu'au début des années 2000, il existait deux catégories d'écoles, celles qui étaient intégralement entretenues par l'État ( fully maintained school») et celles qui, autonomes et de type associatif (« voluntary aided schools»), majoritairement à caractère religieux, recevaient des fonds de l'État mais pour lesquelles environ $10 \%$ des investissements étaient financés par l'Église ou par d'autres organismes d'intérêt public.

Depuis le début des années 2000, ce système scolaire s'est profondément transformé. Un nombre croissant d'écoles financées par l'État sont devenues des academies qui échappent au contrôle des autorités locales. Les academies furent initialement créées par un gouvernement travailliste pour se substituer aux écoles dont on estimait qu'elles avaient échoué et que la qualité de leur enseignement était insuffisante. À leur création, elles devaient être parrainées par des personnes, des entreprises, des associations ou des églises qui établissaient pour les gérer des "trusts» ou fondations ayant le statut d'organisme privé d'intérêt général. Au moment des élections générales de 2010, 203 des 3333 écoles secondaires (soit 
$6 \%$ ) étaient des academies. À l'issue de ces élections, le gouvernement de coalition à dominante conservatrice fit voter la Loi sur les academies de 2010, qui proposait aux autorités locales de "convertir » en academies les écoles restées sous leur contrôle. De nouvelles académies appelées free schools (écoles libres) furent également instaurées, avec l'obligation pour les autorités locales de proposer ce statut pour les établissements à créer pour faire face aux besoins de scolarisation. Il s'ensuivit une rapide expansion, à tel point que les academies et free schools constituaient, en janvier 2019, $32 \%$ des écoles primaires et $75 \%$ des écoles secondaires (DfE, 2019).

\section{LES ACADEMIES}

Les academies sont dirigées par des trusts, fondations privées à but non lucratif auxquelles elles appartiennent et qui, en tant que tels, sont soumis au droit de l'entreprise. Elles sont financées et contrôlées directement par l'État au moyen d'un " accord de financement ", contrat entre le trust de l'academy et le gouvernement central. Pour certains trusts, ce contrat porte sur une seule academy. Pour d'autres, dits trusts multi-academies, un même contrat concerne plusieurs academies. Les écoles appartenant à un trust multi-academies n'ont pas de personnalité juridique et sont gérées directement par le trust; de ce fait, elles jouissent d'une autonomie réduite par rapport aux academies « indépendantes ». En novembre 2017, parmi les 6100 académies du pays, 1668 étaient indépendantes (27\%) et 4432 appartenaient à des trusts multi-academies (73\%) (West and Wolfe, 2019).

L'accord de financement, qui est juridiquement un contrat, définit les obligations des academies. Elles sont tenues d'offrir un curriculum «étendu et équilibré » mais ne sont pas obligées de respecter le Curriculum national, à la différence des écoles qui dépendent des autorités locales. L'accord de financement type en vigueur en 2018 n'impose pas aux academies d'employer des enseignants qualifiés ni de payer leurs enseignants selon les conditions de la convention collectives de l'enseignement scolaire (West and Wolfe, 2019), de sorte qu'elles peuvent s'écarter, à la hausse ou à la baisse, des niveaux de rémunération en vigueur.

Il n'est donc pas surprenant, au vu de ces clauses contractuelles, que la proportion d'enseignants dépourvus de qualification statutaire soit accrue dans les academies par rapport aux écoles relevant des autorités locales. Qui plus est, c'est dans les academies les moins indépendantes, celles qui appartiennent à des trusts multi-academies, que l'on trouve l'accroissement le plus important de cet écart (Martindale, 2019).

En ce qui concerne les clauses financières des accords, le trust d'une academy doit faire certifier ses comptes par un commissaire aux comptes indépendant et les publier au greffe du tribunal de commerce (Company House). Ces comptes, cependant, ne donnent pas le détail des différentes dépenses financées par les deniers publics, contrairement à ce qui est exigé des écoles relevant des autorités locales. En tant qu'organismes indépendants, les trusts ont une autonomie complète pour leurs opérations financières. Bien qu'existent des directives particulières concernant la passation de marchés, les transactions entre personnes apparentées ou les règles de disposition des fonds publics, on a fait état de craintes sur la manière dont les trusts utilisaient l'argent public (West and Wolfe, 2019). Ainsi, la commission des 
comptes publics de la Chambre des Communes (HC PAC, 2018) a-t-elle fait part de ses préoccupations quant aux rémunérations excessives versées par les trusts d'academies, en faisant remarquer que les traitements des directeurs d'academies étaient plus élevés que ceux des directeurs des écoles relevant des autorités locales

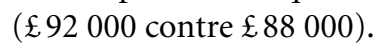

Les trusts d'academies ont besoin d'acheter pour leurs écoles des biens et des services tels que produits et services relatifs aux technologies de l'information et de la communication, internet, photocopies, publications, systèmes téléphoniques, énergie, équipement et services de restauration, assurances GRH et conseils juridiques. À cette occasion, ils peuvent favoriser des personnes ou des entreprises avec lesquelles ils ont des liens personnels ou familiaux, à travers ce que l'on appelle des "transactions entre apparentés ", qui sont une forme de favoritisme. La commission des comptes publics de la Chambre des Communes a relevé, au cours de l'année 2015/2016, trois mille transactions de ce type impliquant les trusts d'academies, pour un montant de cent vingt millions de livres (HC PAC, 2018). Elle faisait observer que certaines d'entre elles n'avaient pas respecté les règles ou suscitaient des doutes quant à leur pertinence (voir également West and Wolfe, 2019).

\section{EXTERNALISATION}

On appelle habituellement externalisation le fait de se procurer, auprès de fournisseurs spécialisés, en général des entreprises du secteur privé, des services auparavant assurés en interne par l'autorité locale ou l'école. Jusqu'au milieu des années 1980, les autorités locales assuraient elles-mêmes, en s'appuyant sur leurs services propres, la plupart des prestations nécessaires aux écoles. À la suite de l'introduction de l'obligation de mettre en concurrence les fournisseurs, elles durent faire des appels d'offre pour de nombreux services qu'elles produisaient. La loi sur le gouvernement local de 1999 généralisa ce processus, avec pour objectif la réduction des coûts des collectivités locales. Dans le cadre défini par la réglementation, une école ou une autorité locale peut donc décider d'externaliser un service en recourant à un appel d'offres.

Un exemple remarquable d'externalisation dans le secteur de l'enseignement scolaire concerne le London Borough de Southwark, où la totalité de l'enseignement fut attribué par contrat à une entreprise privée et où un rapport d'inspection défavorable de l'Ofsted (inspection nationale de l'éducation) contraignit l'État à intervenir pour y mettre fin. En 2001, le London Borough de Southwark avait signé avec la société Atkins Education un contrat de cinq ans lui confiant la fourniture de tous ses services d'enseignement. Ce contrat, d'une valeur de cent millions de livres, incluait un certain nombre d'indicateurs de performance. En 2003, Atkins rencontra des difficultés financières et les indicateurs commencèrent à se dégrader rapidement. La société se résolut à mettre fin au contrat qui était de plus en plus difficile à exécuter sur le plan financier. Le coût de la résolution du contrat se serait élevé à deux millions de livres, principalement en honoraires d'avocats, financé par le gouvernement, l'autorité locale et une contribution limitée d'Atkins (West and Currie, 2008). 
En réponse à des critiques nombreuses et très médiatisées de l'utilisation de l'externalisation par les collectivités, de nombreuses autorités locales ont choisi, à la fin des années 2010, de ré-internaliser certaines fonctions, pour différentes raisons : l'insuffisance des performances des sous-traitants, la nécessité d'étendre leurs capacités dans le cadre d'une restructuration d'ensemble des services ou à l'échéance d'un contrat lorsqu'elles ne souhaitaient pas le renouveler ou lancer un nouvel appel d'offre (Sandford, 2019).

\section{LES PARTENARIATS PUBLIC-PRIVÉ}

L'Initiative de financement privé (Private Finance Initiative), établie en 1992 et abolies en 2018, était un type de contrat de partenariat public-privé (PPP). Les contrats en cours se poursuivent jusqu'à leur échéance. Les modalités de ces contrats sont variables mais ils concernent tous des investissements dans la construction ou la réhabilitation d'un équipement public, comme une école, par un consortium privé, à la suite d'un appel d'offre lancé par une autorité locale. Le contractant s'engage à construire l'école et à fournir, sur la période contractuelle, un ensemble de services comme la maintenance, le chauffage ou la restauration scolaire pour le compte de l'autorité locale. Une fois l'école construite ou rénovée, le contractant est responsable de la maintenance du bâtiment et de la fourniture des services prévus au contrat. L'autorité locale verse au consortium un loyer mensuel pour l'utilisation de l'infrastructure aussi longtemps que les clauses du contrat sont respectées. À la fin du contrat, l'autorité locale retrouve la propriété du bâtiment ou de l'équipement. Initialement, le recours aux partenariats public-privé a été justifié par les besoins de financement des investissements du secteur public soumis à des contraintes imposées par l'État sur les dépenses en capital et sur le recours à l'endettement (McCabe et al., 2001) mais, par la suite, on en vint à le considérer comme un substitut au financement des investissements publics (Lonsdale, 2005).

L'argument économique évoqué en faveur du partenariat public-privé de ce type est qu'il présente le meilleur rapport coût-bénéfice. Pourtant, une étude de la Commission nationale d'audit (2003) sur les partenariats public-privé dans l'enseignement a montré que la qualité des écoles construites dans le cadre d'un PPP était moindre que celle des écoles à maîtrise d'ouvrage publique et qu'aucune différence n'existait entre elles en matière de coûts unitaires en capital et de coûts de maintenance, d'eau, d'assainissement et de combustible. Les coûts de gardiennage et d'entretien se révélaient même supérieurs, en moyenne, dans les écoles en Private Finance Initiative [ou PPP de ce type], peut-être du fait que ces contrats incitent les partenaires privés à fournir une qualité de services supérieure et donc à un coût plus élevé. La commission faisait aussi observer que les clauses type des contrats de Private Finance Initiative (PFI) relatives aux modifications du contrat en cours d'exécution posaient problème en cas de changements fréquents et de faible portée.

Jusqu'en 2018, les PPP furent largement utilisés par l'État et par les collectivités, dont la dépendance à l'égard des partenaires privés apparut de manière éclatante lorsque, en janvier 2018, Carillion, une grande entreprise de construction et de gestion d'équipements, fut mise en liquidation, affectant un grand nombre de contrats passés par des ministères et par des collectivités locales. Selon l'estimation de 
l'Association des collectivités locales, trente conseils municipaux et deux cent vingt écoles ont été directement touchés par l'effondrement de Carillion : quoique principalement entreprise de construction, Carillion fournissait aux écoles de nombreux services tels que la restauration scolaire, l'entretien, le gardiennage ou la maintenance. La Commission de l'administration publique de la Chambre des Communes (2018) remarquait que «l'échec de Carillion reflète les échecs durables de la position du gouvernement sur la conception, la passation et le suivi des contrats et de l'externalisation ». Dans le sillage de l'effondrement de Carillion, le gouvernement a interdit tout nouveau contrat de partenariat public-privé (Davies, 2018).

\section{VERS UNE RÉDUCTION DU CONTRÔLE DÉMOCRATIQUE SUR L'ÉDUCATION}

On peut conclure à une réduction du contrôle démocratique qui s'exerce sur l'éducation en Angleterre du fait de la privatisation de l'enseignement scolaire, des inquiétudes répétées sur l'évolution des coûts et sur le manque de transparence et d'ouverture.

Tout modèle d'enseignement qui s'écarte de la prise en charge directe par les autorités locales soulève des interrogations sur son contrôle démocratique. Les organismes à financement public sont contrôlés par leurs usagers à travers le 82 processus démocratique, alors que les organismes privés doivent, en théorie, rendre des comptes à ceux qui les financent à travers des mécanismes de marché. Dans le système éducatif, la nécessité de rendre des comptes est conceptuellement difficile à saisir et rendue de plus en plus complexe du fait des nouvelles relations qui s'établissent entre les acteurs publics et privés, ainsi que de la privatisation croissante.

Dans le cas des académies, le contrôle n'est pas exercé par l'autorité locale ou par les conseils d'administration comme dans les écoles relevant des autorités locales, du simple fait que la composition des conseils d'administration des trusts est déterminée par les trusts eux-mêmes. En cas de défaillance de leur part, les seuls recours sont entre les mains du gouvernement et de son service d'inspection, l'Ofsted, et, de manière ultime, de celles des tribunaux qui seront saisis des conflits (West et Wolfe, 2019).

En ce qui concerne les partenariats public-privé, les mécanismes de contrôle démocratique sont opaques. Les autorités locales ont la capacité de définir le contenu du contrat et les partenaires privés sont responsables devant elles de son exécution. Cependant, comme la durée du contrat est très longue, les autorités ne peuvent s'adapter aux modifications de la situation qu'au prix d'une coûteuse renégociation. L'autorité peut ainsi se trouver prisonnière du contrat. À ceci s'ajoute l'impossibilité de transférer intégralement le risque au secteur privé dès lors que la scolarité obligatoire donne à l'État la responsabilité d'assurer l'enseignement. Au niveau de chaque école, les conseils d'administration de l'établissement ou du trust d'academy ne peuvent plus prendre de décisions dans les domaines que le contrat a attribués au partenaire, dont le seul interlocuteur est l'autorité signataire du contrat. Si l'autorité locale ne parvient pas à résoudre par la négociation avec le partenaire les problèmes 
soulevés par les écoles, c'est un processus judiciaire, et non un processus démocratique, qui apportera la solution (voir également West et Currie, 2008).

Un autre sujet d'inquiétude porte sur l'absence de transparence et de publicité, en particulier lors de la cession d'écoles détenues par un trust. En 2017, le Wakefield City Academies Trust, qui regroupait vingt-et-une academies, dut se dessaisir de ses établissements en raison de la baisse de niveau de leur enseignement. Quand un trust multi-academies est amené à céder ses écoles, le ministère de l'éducation doit servir d'intermédiaire pour trouver un autre trust qui les reprendra. Cette intermédiation n'implique pas de consultation des parents ni de publicité du processus utilisé. Des complications peuvent aussi apparaître, au cas où une telle cession aboutirait, dans une zone géographique donnée, à priver d'école la population locale. On peut aussi craindre que la défaillance d'un trust mette en péril les ressources financières et matérielles des écoles (HC PAC, 2018).

Au niveau national, les divers types de privatisation de l'enseignement scolaire ont entraîné un coût substantiel pour les finances publiques. Ainsi, quand les autorités locales transforment leurs écoles en academies, le ministère de l'éducation contribue financièrement aux coûts de conversion. Un rapport publié en 2018 par l'Office national d'audit fait état, pour l'année 2016/2017, de dépenses de 81 millions de livres du ministère de l'éducation pour la conversion d'écoles en academies, portant le total des dépenses de conversion depuis 2010/2011 à 745 millions, montant préoccupant pour la bonne utilisation des fonds publics. Les externalisations et les partenariats public-privé sont aussi susceptibles de grever les finances de l'État, notamment à travers les coûts de renégociation et les coûts liés à la rupture des contrats par le contractant privé.

Pour conclure, on peut dire que la privatisation du système éducatif anglais correspond à des configurations variées. Elle apparaît avec le transfert des écoles gérées par les autorités locales à des trusts d'academies privés sans but lucratif, avec les changements que le modèle de l'entreprise privée implique pour le contrôle externe $\mathrm{du}$ fonctionnement des écoles, avec les changements que le recours aux financements privés (PPP) apporte à leur fonctionnement et l'investissement en capital et enfin avec la réduction du rôle des autorités locales due à la création des academies et, de manière plus générale aux politiques d'externalisation. Ces mutations profondes de la nature de l'enseignement scolaire ont tendu à transformer la manière dont on rend des comptes à la population en réduisant le contrôle démocratique et en accroissant les modes indirects de contrôle public que sont les contrats et le recours aux tribunaux. Elles ont aussi affecté la transparence et la publicité de l'action publique et se sont traduites par une charge supplémentaire pour les finances publiques.

\section{RÉFÉRENCES BIBLIOGRAPHIQUES}

AUDIT COMMISSION (2003). PFI in schools: The quality and cost of buildings and services provided by early Private Finance Initiative schemes. London: Audit Commission.

DAVIES R. (2018). « Hammond abolishes PFI contracts for new infrastructure projects ». The Guardian, 29 octobre. [En ligne : https://bit.ly/2PMr6uz].

DEPARTMENT FOR EDUCATION (2019). Schools, pupils and their characteristics: January 2019. London : DfE. [En ligne : https://bit.ly/2Plg7sO]. 
HOUSE OF COMMONS PUBLIC ACCOUNTS COMMITTEE (2018). Converting schools to academies, HC 697, London: House of Commons. [En ligne : https://bit.ly/35oGB2l].

HOUSE OF COMMONS PUBLIC ADMINISTRATION AND CONSTITUTIONAL AFFAIRS COMMITTEE (2018). After Carillion: Public sector outsourcing and contracting. HC 748, London : House of Commons. [En ligne : https://bit.ly/34tGEsw].

LONSDALE C. (2005). «Post-contractual lock-in and the UK Private Finance Initiative (PFI): the cases of national savings and investments and the Lord Chancellor's Department ». Public Administration, 83, 1, p. 67-88.

McCABE B., McKENDRICK J. et KEENAN J. (2001). "PFI in schools - pass or fail? ", Journal of Finance and Management in Public Services, 1, p. 63-74.

NATIONAL AUDIT OFFICE (2018). Converting maintained schools to academies. London : National Audit Office. [En ligne : https://bit.ly/2YN5vWU].

MARTINDALE N. (2019, à paraître). « Does outsourcing school systems degrade education workforces? Evidence from 18,000 English state schools ». British Journal of Sociology of Education.

SANDFORD M. (2019). Local government: alternative models of service delivery. London : House of Commons Library. [En ligne : https://bit.ly/2rS1aWn].

WEST A. et CURRIE P. (2008). « The role of the private sector in publicly-funded schooling in England: Finance, delivery and decision making ». Policy and Politics, 36, 2, p. 191-207.

WEST A. et WOLFE D. (2019). « Academies, autonomy, equality, and democratic accountability: Reforming the fragmented publicly-funded school system in England». London Review of Education, 17, 1, p. 70-86.

WHITFIELD D. (2006). A Typology of Privatisation and Marketisation. European Services Strategy Unit. [En ligne : https://bit.ly/2RTGjMX]. 